\title{
Predicting the host of influenza viruses based on the word vector
}

\author{
Beibei Xu ${ }^{1}$, Zhiying Tan ${ }^{1}$, Kenli Li ${ }^{1}$, Taijiao Jiang ${ }^{\text {Corresp., }}{ }^{2}$, Yousong Peng ${ }^{\text {Corresp. } 3}$ \\ ${ }^{1}$ College of Computer Science and Electronic Engineering, Hunan University, Changsha, China \\ 2 Center of System Medicine, Institute of Basic Medical Sciences, Beijing, China \\ ${ }^{3}$ College of Biology, Hunan University, Changsha, China \\ Corresponding Authors: Taijiao Jiang, Yousong Peng \\ Email address: taijiao@ibms.pumc.edu.cn, pys2013@hnu.edu.cn
}

Newly emerging influenza viruses continue to threaten public health. A rapid determination of the host range of newly discovered influenza viruses would assist in early assessment of their risk. Here, we attempted to predict the host of influenza viruses using the Support Vector Machine (SVM) classifier based on the word vector, a new representation and feature extraction method for biological sequences. The results show that the length of word within the word vector, the sequence type (DNA or protein) and the species from which the sequences were derived for generating the word vector all influence the performance of models in predicting the host of influenza viruses. In nearly all cases, the models built on the surface proteins hemagglutinin (HA) and neuraminidase (NA) (or their genes) produced better results than internal influenza proteins (or their genes). The best performance was achieved when the model was built on the HA gene based on word vectors (words of three-letters long) generated from DNA sequences of the influenza virus. This results in accuracies of $99.7 \%$ for avian, $96.9 \%$ for human and $90.6 \%$ for swine influenza viruses. Compared to the method of sequence homology best-hit searches using Basic Local Alignment Search Tool (BLAST), the word vector-based models still need further improvements in predicting the host of influenza A viruses. 
1 Predicting the host of influenza viruses based on the word vector

2 Beibei Xu ${ }^{1}$, Zhiying Tan ${ }^{1}$, Kenli $\mathrm{Li}^{1}$, Taijiao Jiang, ${ }^{2,3}$, , Yousong Peng4,*

3

$4{ }^{1}$ College of Computer Science and Electronic Engineering, Hunan University, Changsha, 410082, China

$5 \quad{ }^{2}$ Center of System Medicine, Institute of Basic Medical Sciences, Chinese Academy of Medical Sciences

6 \& Peking Union Medical College, Beijing, 100005, China

$7 \quad{ }^{3}$ Suzhou Institute of Systems Medicine, Suzhou, Jiangsu, 215123, China

$8 \quad{ }^{4}$ College of Biology, Hunan University, Changsha, 410082, China

$10 *$ To whom correspondence should be addressed. Email: taijiao@moon.ibp.ac.cn(TJ) or

11 pys2013@hnu.edu.cn(YP)

13 Abstract

14 Newly emerging influenza viruses continue to threaten public health. A rapid

15 determination of the host range of newly discovered influenza viruses would assist

16 in early assessment of their risk. Here, we attempted to predict the host of

17 influenza viruses using the Support Vector Machine (SVM) classifier based on the

18 word vector, a new representation and feature extraction method for biological 
19 sequences. The results show that the length of word within the word vector, the

20 sequence type (DNA or protein) and the species from which the sequences were

21 derived for generating the word vector all influence the performance of models in

22 predicting the host of influenza viruses. In nearly all cases, the models built on the

23 surface proteins hemagglutinin (HA) and neuraminidase (NA) (or their genes)

24 produced better results than internal influenza proteins (or their genes). The best

25 performance was achieved when the model was built on the HA gene based on

26 word vectors (words of three-letters long) generated from DNA sequences of the

27 influenza virus. This results in accuracies of $99.7 \%$ for avian, $96.9 \%$ for human

28 and $90.6 \%$ for swine influenza viruses. Compared to the method of sequence

29 homology best-hit searches using Basic Local Alignment Search Tool (BLAST),

30 the word vector-based models still need further improvements in predicting the

31 host of influenza A viruses.

33 Introduction

34 The influenza virus is a negative-sense, single-stranded, segmented RNA virus. Its

35 genome is composed of eight segments and mainly encodes twelve proteins,

36 including two surface proteins HA and NA, and ten internal proteins PB2, PB1, PA,

37 NP, M1, M2, NS1, NS2, PA-X and PB1-F2. Influenza viruses could be mainly 
separated into types A, B and C, while type A could be further separated into subtypes according to the HA and NA proteins, such as $\mathrm{H} 3 \mathrm{~N} 2, \mathrm{H} 1 \mathrm{~N} 1, \mathrm{H} 5 \mathrm{~N} 1$, and so on (Taubenberger \& Kash 2010). Type B and C influenza viruses mainly infect humans, whereas type A can infect a wide range of species, such as birds (poultry) and mammals (pigs, bats) including humans (Webster et al. 1992). Among them, avian, human and swine influenza viruses are most commonly observed, and cause large health and economic loss to human society. In recent years, human infections by what were considered typical avian and swine strains have become more common, for instance infections due to influenza H7N9 and H5N8 (Peiris et al. 2016; Su et al. 2015). Rapid determination of the host range of a given influenza virus could assist in early evaluation of the potential risk of emerging subtypes.

Avian influenza virus is considered to be the evolutionary ancestor of all other influenza viruses (Webster et al. 1992). When an avian influenza virus infects a different host species and is able to spread within this new host, mutations rapidly accumulate as the viral population adapts to this host. This situation has been observed in human and swine influenza viruses. Several molecular markers for human or other influenza viruses have been identified at the amino acid level, either experimentally or by means of computational analyses (Chen et al. 2006; Finkelstein et al. 2007; Kim et al. 2010; Tamuri et al. 2009). For example, a Lysine in position 627 of internal protein PB2 tends to be favored in human strains, while 
58 avian strains usually have a Glutamic acid at this position (Finkelstein et al. 2007;

59 Kim et al. 2010). Several studies further attempted to classify the host of influenza

60 virus by machine learning methods (ElHefnawi \& Sherif 2014; Sherif et al. 2011;

61 Attaluri et al. 2010). For example, Attaluri et al. integrated multiple machine

62 learning techniques to predict the host of influenza A viruses and achieved

63 accuracies ranging from 0.84 to 0.98 . However, most of these studies either used a

64 selection of HA subtypes only, or a relatively small dataset, ignoring the real,

65 extensive genetic diversity of influenza viruses.

66 In this work, we used the largest influenza dataset known to date, including 163666

67 unique DNA and 150947 unique protein sequences, to predict the host of influenza

68 viruses based on the nucleotide and amino acid word vector. The word vector is a

69 new representation and feature extraction method (Mikolov et al. 2013), which was

70 originally developed and used in natural language processing. It was first applied

71 to biological research in 2015 (Asgari \& Mofrad 2015) and proved to be useful for

72 protein family classification and disordered protein prediction. Here, we applied

73 word vectors to predict the host of influenza viruses and achieved an overall

74 accuracy of 0.97 , which further demonstrated its strength in biological sequence

75 representation. 
77 Materials and Methods

78 Overview of this work

79 Figure 1 shows the workflow of this work. Firstly, all protein and DNA sequences

80 of influenza viruses (denoted as influenza protein and DNA dataset respectively)

81 and all non-redundant protein sequences of all species (denoted as SwissProt

82 dataset) were collected, which were used to generate word vectors by the tool

83 word2vec. Then, all non-redundant protein and DNA sequences of influenza A

84 viruses with known host (avian, human and swine) were transformed into protein

85 and DNA vectors based on word vectors. Finally, the Support Vector Machine

86 (SVM) models were built for classifying influenza viruses of avian/human,

$87 \mathrm{avian} /$ swine and human/swine based on protein or DNA vectors. A voting strategy

88 was used to determine the predicted host for the influenza virus.

\section{Datasets}

90 The SwissProt dataset were derived from the Swiss-Prot database on UniProt

91 (UniProt 2016) on April 11 $1^{\text {th }}, 2016$. It contains 550740 protein sequences with a

92 length ranging from 11 to 35213 amino acids (aa).

93 For the influenza DNA dataset, the nucleotide (nt) sequences for eight genes, 94 including HA and NA as well as internal protein genes PB2, PB1, PA, NP, MP and 
NS, of influenza A viruses with known host (avian, human and swine) were extracted from the database of Influenza Virus Resources (Bao et al. 2008) on April 26th, 2016. At the same time, amino acid sequences of the 12 proteins (HA and NA, and the ten internal proteins) were extracted to produce an influenza protein dataset. In total, 385788 DNA and 607327 protein sequences were collected. To reduce the computational cost, redundancy was removed at $100 \%$ level with the help of the software package cd-hit (Li \& Godzik 2006). This step maintained 163666 unique DNA and 150947 unique protein sequences for analysis. The number of protein sequences used in this study for each protein of avian, human and swine influenza viruses is shown in Table 1; the number of genes is included in Table S1. These datasets are much larger than those used in previous studies.

\section{Word vector generation and protein sequence vectorization}

The tool word2vec is a software package developed for producing word embeddings (Mikolov et al. 2013). It takes a large corpus of text (here, it refers to large number of protein or DNA sequences which were separated into words) as its input and outputs a vector space of several hundred dimensions. Each unique word in the texts is assigned a corresponding vector in the space, during which they are closely located to other words that share a common context. Here, the tool word2vec was used to generate the word vectors of 200 dimensions using the 
115 SwissProt dataset and the influenza datasets respectively. The skip-gram model

116 and hierarchical softmax algorithm were used in the word2vec, with other

117 parameters in default values. The word vectors with words of two to four amino

118 acids (or nucleotides) long were all generated in the same way.

119 The vectorization of protein (or DNA) sequences was adapted from Asgari and

120 Mofrad's work (Asgari \& Mofrad 2015). Firstly, each protein (or DNA) sequence

121 was separated into overlapping words of $\mathrm{N}(2 \sim 4)$ amino acids (or nucleotides).

122 Then, the word vectors for all these words were summed up and averaged, which

123 led to the protein (or DNA) vectors of 200-dimensions for the protein (or DNA)

124 sequences.

\section{Predicting the host for the influenza virus with SVM}

The SVM models for predicting the host of influenza viruses were built using functions of svmtrain() and svmclassify() (Change \& Lin 2011) in MATLAB R2014b. The Gaussian Radial Basis Function kernel "rbf" with default parameters was used for the SVM models. Three SVM models were built to discriminate the influenza viruses of avian and human, avian and swine, human and swine based on word vectors. A simple voting strategy was used to determine the final prediction for the host of influenza viruses. Ten-fold cross-validations were used to evaluate the performance of the SVM models. 
135 The method of Profile hidden Markov model (HMM) through the package of 136 HMMER3 (Eddy 2010), and the method of Basic Local Alignment Search Tool 137 (BLAST) through the package of BLAST+ (Altschul et al. 1990), were used for 138 inferring the host of influenza A viruses based on homologies of protein or DNA 139 sequences. For each gene or protein, $75 \%$ of protein (or DNA) sequences were 140 randomly selected for building the library (for BLAST) or profile (for HMM), 141 while the remaining protein (or DNA) sequences were used to test through the best 142 hit search.

Results

\section{Predict the host of influenza viruses based on word vectors derived from} influenza protein dataset

We firstly attempted to predict the host of influenza A viruses based on word 147 vectors derived from influenza protein dataset. Figure 2 shows that the SVM 148 models built on the surface proteins HA and NA, and on the internal proteins PB2, PB1, PA and NP, performed much better than those built on other internal proteins did (including M1, M2, NS1, NS2, PB1-F2 and PA-X). The overall accuracies

151 ranged from 0.79 0.96 (summarized in Table S2). The length of word in the word 152 vector has a significant influence on the model's performances: the models based 
153 on four-letters words performed best for all proteins. Further analyses on the 154 model's performance by host show that all models predict most accurately for the 155 avian influenza virus, with accuracies ranging from $0.97 \sim 1$. For human and swine 156 influenza viruses, the models achieved accuracies of approximately 0.9 for HA, 157 NA, PB2, PB1, PA and NP, while for the other proteins performance was rather 158 poor.

159

160

\section{Predict the host of influenza viruses based on word vectors derived from the} SwissProt dataset

We next investigated the influence of species of protein sequence, which were used to generate the word vector, on the prediction of the host of influenza viruses. The SwissProt dataset included protein sequences of virus, bacteria, fungi, plant, animal, and so on. In theory, the word vectors derived from the influenza protein dataset should reflect more accurately the influenza virus than those derived from the SwissProt dataset. Figure 3 shows the overall accuracies for the SVM models based on two kinds of word vectors with words of two to four amino acids long. The models based on two kinds of word vectors achieved comparable performances. For the word vector with words of two to three letters long, the models based on word vectors derived from the SwissProt dataset even outperformed those based on word vectors derived from the influenza protein dataset. However, the best performance (overall accuracy greater than 0.96) was 
173 achieved on the model built on the HA protein, which used word vectors with

174 words of four-letters long derived from the influenza protein dataset (Table S2 and

175 S3). Besides, accuracies of models based on word vectors derived from the

176 SwissProt dataset decreased as the length of the word in the word vector, while the

177 opposite trend was observed for models based on those derived from the influenza

178 protein dataset (Figure 3).

179

180

181

182

183

184

185

186

187

188

189

190

191

\section{Predict the host of influenza viruses based on word vectors derived from influenza DNA dataset}

Then, we continued to investigate the influence of data type, DNA or protein, on predicting the host of influenza viruses based on the word vector. The influenza DNA dataset were used to generate word vectors with words of two to four nucleotides long. Figure 4 shows that in most cases, models based on word vectors derived from DNA sequences outperformed those based on word vectors derived from protein sequences. Excellent performance was obtained with a DNA word length of three, with overall accuracies greater than 0.95 for most genes (Table S4). As before, the best performance was achieved on the model based on word vectors of HA gene, with an overall prediction accuracy of 0.97 . More specifically, the prediction accuracy for avian, human and swine influenza viruses equaled 0.997, 0.969 and 0.906 , respectively (Table S4). 
192 Predict the host of influenza viruses based on sequence homology search

193 Sequence homology search through the methods of BLAST and HMM can also be 194 used for inferring the host of viruses. Here, we tested both methods in inferring the 195 host of influenza A viruses based on both protein and DNA sequences. As shown 196 in Table 2, when using the protein sequence, the method based on word vectors 197 outperformed those based on HMM and BLAST, at least for HA, NA, and the 198 proteins PB2, PB1, PA, NP and M2. For the other internal proteins (including the short proteins) the reverse was the case. For the methods based on sequence homology search, BLAST performed slightly better than HMM did, especially for HA and NA. Surprisingly, when using the DNA sequence, the method based on BLAST outperformed all the other methods for nearly all genes (Table S5). It achieved an overall accuracy of 0.979 on the HA gene, which is greater than that of 204 all the other models tested here.

\section{Discussion}

This work investigated the prediction of the host of influenza A viruses based on word vectors. For all genes or proteins, the predictions for avian influenza viruses were more accurate than for human or swine influenza viruses. This may partly be caused by occasional infections of humans or swine by what actually were avian

210 influenza viruses (Beigel et al. 2005; Claas et al. 1998), which may have weakened 
211 any host-specific signal in the non-avian hosts.

212 The surface proteins HA and NA were observed to be better discriminators for the

213 host of influenza viruses than the internal viral proteins. This is most likely related

214 to the selective pressure posed by the host: since HA and NA are the main antigens

215 of influenza virus they will be recognized by the immune system of the host

216 (Couch \& Kasel 1983). Therefore, these proteins have to mutate rapidly to

217 maintain a stable population in a new host, a mechanism of host adaptation that

218 will lead to divergence of lineages. Surprisingly, the results obtained with internal

219 proteins PB2, PB1, PA and NP were comparable to those of the surface proteins.

220 These proteins constitute the RNA polymerase complex of the virus, which is

221 responsible for RNA replication and is thus directly responsible for the survivor

222 ability of the virus (te Velthuis \& Fodor 2016). All viral proteins are translated by

223 the host, but these proteins are most important for rapid viral reproduction, thus

224 they will also adapt rapidly to the new host (Mehle \& Doudna 2009).

225 As it is known, the HA proteins diverge most among all the proteins of influenza A

226 viruses, which results in 18 HA subtypes reported until now (Tong et al. 2013).

227 The best performance achieved on the model of HA protein suggests that the word

228 vector may capture the intrinsic difference between the influenza virus of different

229 hosts, irrespective of the HA subtypes. 
230 The word vectors could be generated with protein or DNA sequences of any

231 species. In theory, the word vector derived from the influenza protein dataset could

232 reflect more accuracy the influenza virus than those derived from the SwissProt

233 dataset. However, in most cases, the models based on the former performed

234 comparably with those based on the latter (Figure 3). This may reflect the similar

235 principles in organizing amino acids into protein sequences in all species.

236 A limitation of this work is that only the word vector was used in predicting the

237 host of influenza viruses. More features such as the amino acids composition,

238 motif frequency and molecular markers may be integrated to improve the accuracy

239 of models in predicting the human and swine influenza viruses. Overall, this work

240 should be an interesting attempt in using the word vector in biological sequence

241 representation. The models based on word vectors achieved high accuracies in

242 predicting the host of influenza viruses, which may be helpful in influenza

243 prevention.

\section{References}

Altschul SF, Gish W, Miller W, Myers EW, and Lipman DJ. 1990. Basic local alignment search tool. J Mol Biol 215:403-410.

Asgari E, and Mofrad MR. 2015. Continuous Distributed Representation of Biological Sequences for Deep 
Attaluri PK, Chen ZX, Lu GQ. 2010. Applying neural networks to classify influenza virus antigenic types and hosts. 2010 IEEE Symposium on Computational Intelligence in Bioinformatics and Computational Biology (CIBCB): IEEE.

Bao YM, Bolotov P, Dernovoy D, Kiryutin B, Zaslavsky L, Tatusova T, Ostell J, and Lipman D. 2008. The influenza virus resource at the national center for biotechnology information. $J$ Virol 82:596-601. Doi 10.1128/Jvi.02005-07

Beigel JH, Farrar J, Han AM, Hayden FG, Hyer R, de Jong MD, Lochindarat S, Nguyen TK, Nguyen TH, Tran TH, Nicoll A, Touch S, and Yuen KY. 2005. Avian influenza A (H5N1) infection in humans. N Engl J Med 353:1374-1385. 10.1056/NEJMra052211

Chang CC and Lin CJ. 2011. LIBSVM: a library for support vector machines. ACM Transactions on Intelligent Systems and Technology, 2:27:1--27:27.

Chen GW, Chang SC, Mok CK, Lo YL, Kung YN, Huang JH, Shih YH, Wang JY, Chiang C, Chen CJ, and Shih SR. 2006. Genomic signatures of human versus avian influenza A viruses. Emerging Infectious Diseases 12:1353-1360. 10.3201/eid1209.060276

Claas EC, Osterhaus AD, van Beek R, De Jong JC, Rimmelzwaan GF, Senne DA, Krauss S, Shortridge KF, and Webster RG. 1998. Human influenza A H5N1 virus related to a highly pathogenic avian influenza virus. Lancet 351:472-477. 10.1016/S0140-6736(97)11212-0

Couch RB, and Kasel JA. 1983. Immunity to influenza in man. Annu Rev Microbiol 37:529-549. 10.1146/annurev.mi.37.100183.002525

ElHefnawi M, and Sherif FF. 2014. Accurate classification and hemagglutinin amino acid signatures for influenza A virus host-origin association and subtyping. Virology 449:328-338. 10.1016/j.virol.2013.11.010

Eddy S. 2010. HMMER3: a new generation of sequence homology search software. Available at http://hmmer.org/.

Finkelstein DB, Mukatira S, Mehta PK, Obenauer JC, Su X, Webster RG, and Naeve CW. 2007. Persistent host markers in pandemic and H5N1 influenza viruses. $J$ Virol 81:10292-10299. 10.1128/JVI.00921-07

Kim JH, Hatta M, Watanabe S, Neumann G, Watanabe T, and Kawaoka Y. 2010. Role of host-specific amino acids in the pathogenicity of avian H5N1 influenza viruses in mice. $J$ Gen Virol 91:1284-1289. 10.1099/vir.0.018143-0

Li W, and Godzik A. 2006. Cd-hit: a fast program for clustering and comparing large sets of protein or nucleotide 
sequences. Bioinformatics 22:1658-1659. 10.1093/bioinformatics/btl158

Mehle A, and Doudna JA. 2009. Adaptive strategies of the influenza virus polymerase for replication in humans. Proc Natl Acad Sci U S A 106:21312-21316. 10.1073/pnas.0911915106

Mikolov T, Chen K, Corrado GS, Dean J. 2013. Efficient Estimation of Word Representations in Vector Space. In Proceedings of Workshop at ICLR, 2013.

Peiris JS, Cowling BJ, Wu JT, Feng L, Guan Y, Yu H, and Leung GM. 2016. Interventions to reduce zoonotic and pandemic risks from avian influenza in Asia. Lancet Infectious Diseases 16:252-258. 10.1016/S1473$3099(15) 00502-2$

Sherif FF, Kadah Y, El-Hefnawi M. 2011. Classification of human vs. non-human, and subtyping of human influenza viral strains using Profile Hidden Markov Models. 2011. 1st Middle East Conference on Biomedical Engineering (MECBME): IEEE.

Su S, Bi Y, Wong G, Gray GC, Gao GF, and Li S. 2015. Epidemiology, Evolution, and Recent Outbreaks of Avian Influenza Virus in China. J Virol 89:8671-8676. 10.1128/JVI.01034-15

Tamuri AU, Dos Reis M, Hay AJ, and Goldstein RA. 2009. Identifying changes in selective constraints: host shifts in influenza. PLoS Comput Biol 5:e1000564. 10.1371/journal.pcbi.1000564

Taubenberger JK, and Kash JC. 2010. Influenza virus evolution, host adaptation, and pandemic formation. Cell Host Microbe 7:440-451. 10.1016/j.chom.2010.05.009

te Velthuis AJW, and Fodor E. 2016. Influenza virus RNA polymerase: insights into the mechanisms of viral RNA synthesis. Nature Reviews Microbiology 14:479-493. 10.1038/nrmicro.2016.87

Tong SX, Zhu XY, Li Y, Shi M, Zhang J, Bourgeois M, Yang H, Chen XF, Recuenco S, Gomez J, Chen LM, Johnson A, Tao Y, Dreyfus C, Yu WL, McBride R, Carney PJ, Gilbert AT, Chang J, Guo Z, Davis CT, Paulson JC, Stevens J, Rupprecht CE, Holmes EC, Wilson IA, and Donis RO. 2013. New World Bats Harbor Diverse Influenza A Viruses. PLoS Pathog 9. ARTN e100365710.1371/journal.ppat.1003657

UniProt. 2016. Available at http://www.uniprot.org/.

Webster RG, Bean WJ, Gorman OT, Chambers TM, and Kawaoka Y. 1992. Evolution and ecology of influenza A viruses. Microbiol Rev 56:152-179. 


\section{Figure Legends}

307 Table 1 The number of non-redundant sequences used in this study for each 308 protein of avian, human and swine influenza A viruses.

\begin{tabular}{|c|c|c|c|c|c|c|c|}
\hline Protein & Avian & Human & Swine & Protein & Avian & Human & Swine \\
\hline HA & 15328 & 17872 & 7132 & M1 & 1717 & 1134 & 1011 \\
\hline NA & 10295 & 9834 & 4802 & M2 & 2360 & 1456 & 1431 \\
\hline PB2 & 8134 & 4363 & 2386 & NS1 & 5364 & 3128 & 2076 \\
\hline PB1 & 7323 & 4026 & 2343 & NS2 & 2231 & 1000 & 995 \\
\hline PA & 7925 & 4173 & 2461 & PB1-F2 & 4151 & 1180 & 934 \\
\hline NP & 4816 & 2261 & 1929 & PA-X & 1716 & 738 & 922 \\
\hline
\end{tabular}

310 Table 2 Comparison of methods for predicting the host of influenza A viruses

311 based on the word vector and based on sequence homology best-hit searches using 312 protein sequences. The table listed the overall accuracies for predicting the host of 313 influenza A viruses. For the method based on word vectors, the optimal model for 314 individual proteins was used. 


\begin{tabular}{|c|c|c|c|}
\hline \multirow{2}{*}{ Protein } & \multicolumn{3}{|c|}{ Methods for predicting the host of influenza A virus } \\
\cline { 2 - 4 } & Word Vector & BLAST & HMM \\
\hline HA & 0.964 & 0.950 & 0.676 \\
\hline NA & 0.955 & 0.914 & 0.593 \\
\hline PB2 & 0.931 & 0.892 & 0.885 \\
\hline PB1 & 0.928 & 0.898 & 0.798 \\
\hline PA & 0.933 & 0.917 & 0.822 \\
\hline NP & 0.912 & 0.837 & 0.830 \\
\hline M1 & 0.712 & 0.676 & 0.672 \\
\hline M2 & 0.509 & 0.807 & 0.867 \\
\hline NS1 & 0.799 & 0.864 & 0.895 \\
\hline NS2 & 0.561 & 0.748 & 0.866 \\
\hline PB1-F2 & 0.712 & 0.952 & 0.955 \\
\hline PA-X & 0.625 & 0.730 \\
\hline
\end{tabular}

316 Figure 1 The workflow of the methodological approach used. For explanation see 317 text. 


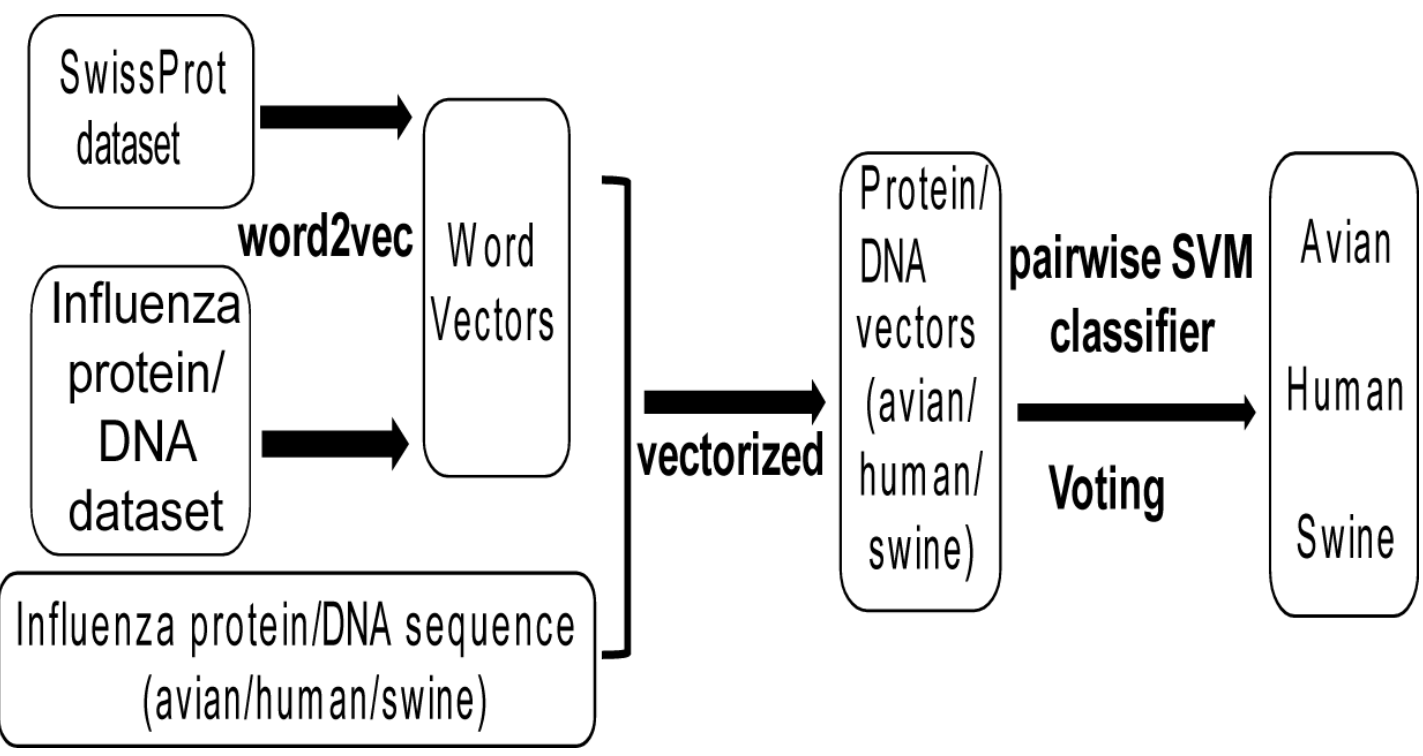

Figure 2 Performances for the models based on the word vector with words of two

323 to four letters long (shown in circle, triangle and star, respectively) derived from

324 the influenza protein dataset. In black the overall prediction accuracy is shown,

325 while red, purple and blue lines represent the accuracies for avian, human and

326 swine influenza viruses, respectively. The accuracies are averaged in ten-fold

327 cross-validations. 


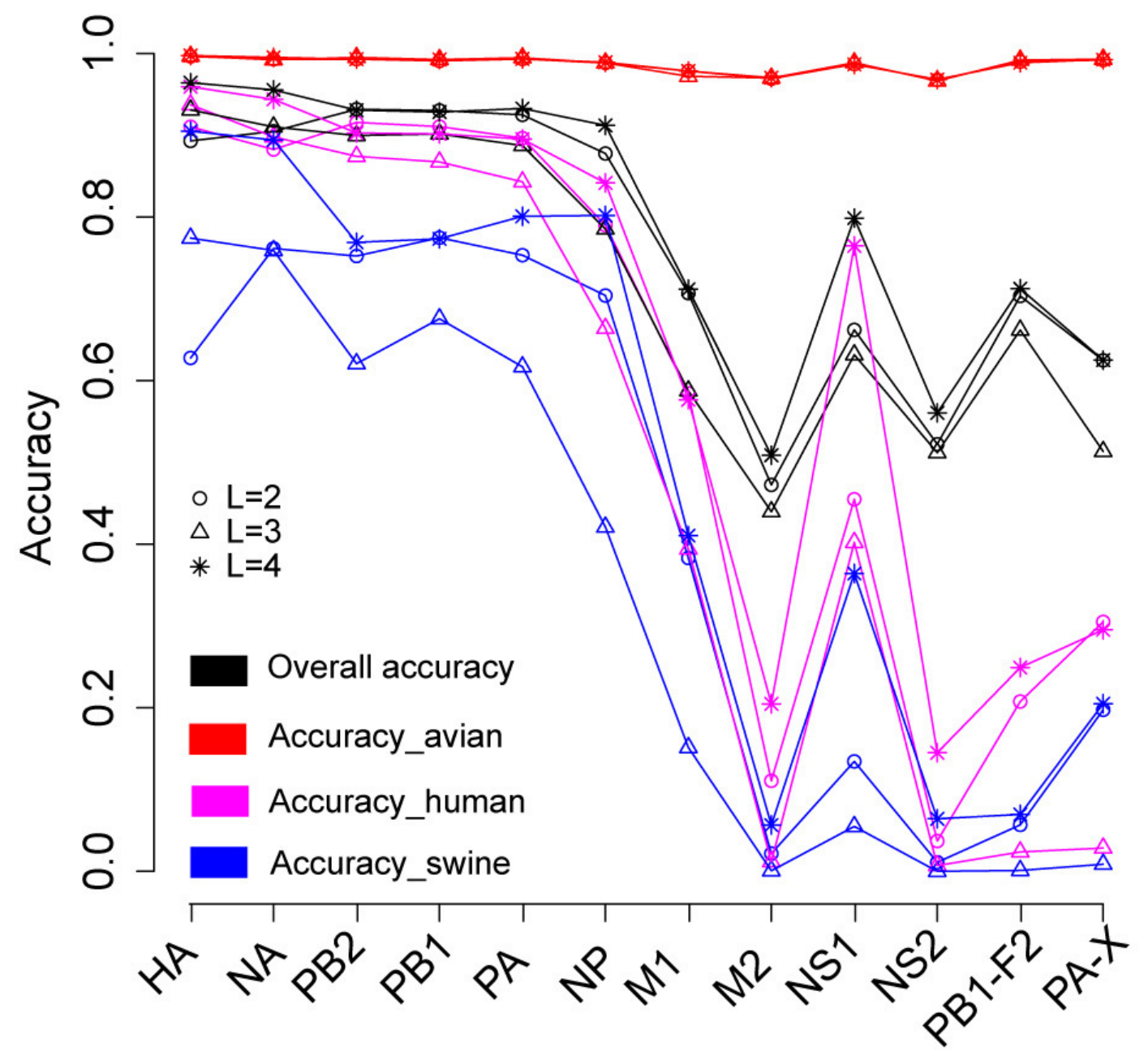


Figure 3 Comparison of overall accuracies for the models based on word vectors

337 with words of two to four letters long (shown in circle, triangle and star,

338 respectively) derived from the influenza protein dataset (red line) and SwissProt

339 dataset (blue line). The accuracies were averaged in ten-fold cross-validations.

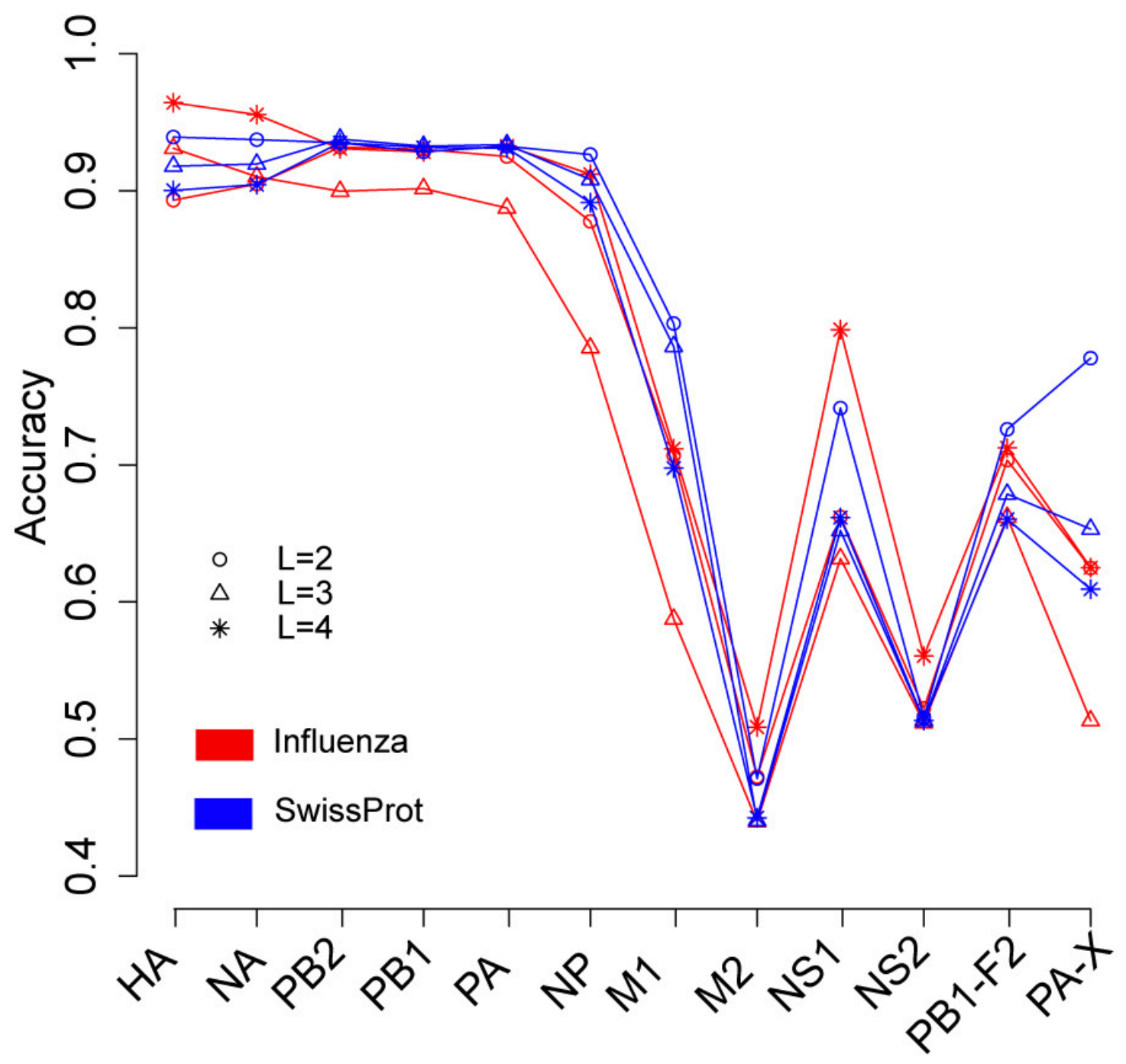


Figure 4 Comparison of overall accuracies for the models based on word vectors

344 with words of two to four letters long (shown in circle, triangle and star,

345 respectively) derived from the influenza DNA dataset (red line) and influenza

346 protein dataset (blue line). The accuracies are averaged in ten-fold cross-

347 validations.

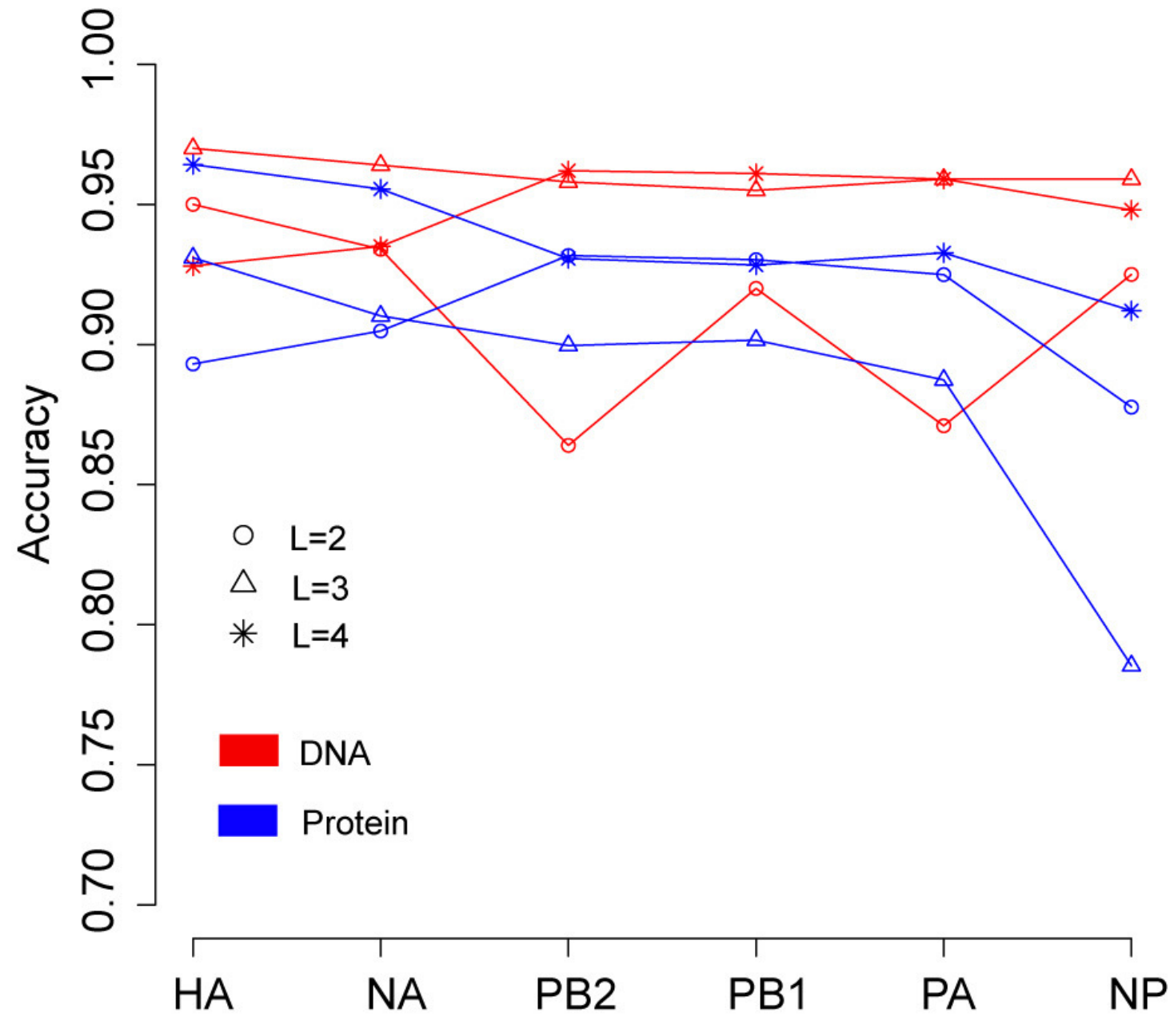

